03

\title{
Слабая оптическая анизотропия и ее обнаружение на примере тетрабората стронция, активированного ионами иттербия
}

\author{
(C) T.X. Хасанов
}

Институт фризики полупроводников Сибирского отделения РАН, лаборатория оптических материалов и структур, 630090 Новосибирск, Россия

e-mail: hasanov@isp.nsc.ru

Поступила в редакцию 29.11.2018 г.

В окончательной редакции 13.02.2019 г.

Принята к публикации 22.02.2019 г.

\begin{abstract}
Предложен и экспериментально реализован метод, позволяющий обнаружить и измерить слабую оптическую анизотропию. Исследован тетраборат стронция, активированный ионами иттербия, который считается аморфным стеклом; в нем обнаружено и измерено наличие анизотропии, соизмеримой с инструментальными погрешностями угломерных устройств эллипсометра. В основе предложенного подхода лежит ранее развитый эффективный и относительно простой метод, для которого вклад в формирование результирующего пучка от поверхностного (переходного) слоя существенно меньше, чем от объема материала. Также используются соотношения измерительных зон эллипсометра. Изложены некоторые важные аспекты оптической и механической юстировки эллипсометров применительно к исследованию слабой анизотропии.
\end{abstract}

Ключевые слова: оптическая анизотропия, тетраборат стронция, эллипсометр.

DOI: $10.21883 /$ OS.2019.08.48040.352-18

\section{Введение}

В настоящее время в оптоэлектронике широко используются различные полупроводниковые и другие структуры, которые обладают оптической анизотропией. Например, двуокись кремния $\left(\mathrm{SiO}_{2}\right)$ имеет более 10 аллотропических модификаций, и все полиморфные модификации этого вещества (кроме $\beta$-кристобалита) анизотропные. Хорошо известно, что аморфная модификация двуокиси кремния $\left(\mathrm{SiO}_{2}\right)$ является ключевым диэлектриком в современных кремниевых и волноводных структурах. Одна из самых распространенных и хорошо изученных модификаций этого вещества $-\alpha$-кварц - имеет гексагональную кристаллическую структуру и обладает слабой оптической анизотропией. Он является не только важным в радиоэлектронике, но и одним из основных элементов в поляризационной оптике [1].

В последнее время все чаще применяются тонкие пленки, получаемые с наклонным осаждением [2]. Пленки $\mathrm{SiO}_{x}$, получаемые наклонным осаждением, также обладают оптической анизотропией [2]. Оптические и фотоэлектрические свойства пленок, получаемые наклонным осаждением, могут существенно меняться в процессе изготовления. Надо отметить, что они имеют колончатую микроструктуру, поэтому обладают двухосной оптической анизотропией. Как утверждается в работе [2], подобные тонкие пленки находят применение в качестве элементов интегральных фотонных систем, узкополосных поляризационных фильтров, фазовых элементов, солнечных батарей. Выбором угла осаждения и других условий осаждения достигаются необходимые качества и область применения. При этом главные показатели преломления могут быть целенаправленно выбраны из непрерывного диапазона значений.

Для повышения эффективности твердотельных источников излучения (оптических квантовых генераторов) в последнее время наметилась тенденция на поиск новых стеклянных матриц (силикатных, фосфатных, боратных и других) различного состава $[3,4]$. Одной из таких сред, перспективной для твердотельных лазеров, является стронций-боратное стекло, активированное ионами иттербия - $\mathrm{Yb}: \mathrm{SrB}_{4} \mathrm{O}_{7}$ (Yb : SBO). Это очень простое по составу практически бессвильное стекло с температурой стеклования в области $1100^{\circ} \mathrm{C}$. Во всех вышеперечисленных объектах часто возникает необходимость экспресс-оценки наличия оптической анизотропии и измерения их оптических характеристик.

Следует напомнить о таких материалах, как нитрид галлия и танталат лития. Эти материалы также обладают слабой оптической анизотропией и имеют широкое применение. Например, спектр применения в оптоэлектронике нитрида галлия действительно широкий: светодиоды сине-зеленой области видимого спектра, ближнего ультрафиолетового диапазона, активные среды лазерных диодов и др. Также структуры на основе нитрида галлия перспективны не только в оптоэлектронике, но и для разработки компонентной базы силовой и СВЧ-электроники. Гетероструктура на основе нитрида галлия обладает оптической анизотропией $-\Delta n \sim 3 \cdot 10^{-3}[5,6]$.

Наиболее эффективным и неразрушающим средством исследования подобных структур является метод эллипсометрии. Как показано в работе [2], для определения всех трех главных компонентов тензора диэлектриче- 
ской проницаемости $\varepsilon$ и угла наклона осей при использовании одних лишь угловых зависимостей эллипсометрических параметров необходимо обеспечить погрешность измерения не более $0.0001^{\circ}$, что находится далеко за пределами точности, обеспечиваемой современными эллипсометрами.

Целью настоящей работы является разработка и обоснование новых эллипсометрических подходов для обнаружения и измерения слабой оптической анизотропии. Развивается ранее предложенная методика для исследования объемных свойств отражающих и пропускающих систем [7-9]. Кроме того, используются соотношения измерительных зон эллипсометра для изотропных и анизотропных систем [10,11]. Излагаются некоторые важные аспекты оптической и механической юстировки эллипсометров применительно к исследованию слабой анизотропии. Исследован тетраборат стронция, активированный ионами иттербия, который считается аморфным стеклом, и в котором обнаружена и измерена анизотропия, соизмеримая с инструментальными погрешностями угломерных устройств эллипсометра. Экспериментальная реализация цели достигнута на модифицированном эллипсометре ЛЭФ-2.

\section{Эллипсометрия}

При взаимодействии света с объектом исследования происходит зеркальное отражение, преломление, рассеяние и изменение состояния эллипса поляризации. Последнее весьма чувствительно к изменениям оптических параметров исследуемого объекта и является основой эллипсометрии.

Как было ранее отмечено, закон взаимодействия падающей волны с оптической системой можно представить в виде простого линейного преобразования вектора Джонса этой волны $[9,10]$ :

$$
E_{0}=T E_{i}
$$

где $E_{0}, E_{i}$ - комплексные амплитуды до и после взаимодействия световой волны с оптической системой, $T$ - матрица Джонса для оптической системы. Для отражающей системы матрица Джонса $T$ эквивалентна матрице отражения $R[9,10]$ :

$$
R=\left|\begin{array}{ll}
R_{p p} & R_{p s} \\
R_{s p} & R_{s s}
\end{array}\right|
$$

Здесь $R_{p p}, R_{s s}$ - комплексные коэффициенты отражения для света, поляризованного в плоскости падения ( $p$-направление) и перпендикулярно ей ( $s$-направление), $R_{p s}, R_{s p}$ - недиагональные элементы матрицы Джонca, обусловленные анизотропией отражающей системы. При отражении от любой многослойной изотропной отражающей системы или анизотропной, но с симметричными ориентациями недиагональные элементы $R_{p s}$,
$R_{s p}$ равняются нулю. Напомним, что параметр

$$
\rho=\frac{R_{p p}}{R_{s s}}=\operatorname{tg} \Psi e^{i \Delta}
$$

представляет собой ту величину, которая описывает поляризацию света после отражения от изотропной, в общем случае многослойной системы. Углы $\Psi$ и $\Delta$, характеризующие относительный коэффициент отражения, обычно называют поляризационными углами отражающей системы или эллипсометрическими параметрами, а уравнение (1) - основным уравнением эллипсометрии $[9,10]$. При одном угле падения света на образец, измеряя эллипсометрические углы $\Psi, \Delta$ и решая уравнение (1), можно определить два любых неизвестных параметра отражающей системы. В обобщенной эллипсометрии имеются три комплексные уравнения [9-11]. Эти комплексные уравнения независимы, следовательно, при одном угле падения возможно определение до шести параметров отражающей системы. Однако в этом случае решение как прямой, так и обратной задач эллипсометрии громоздкое и трудоемкое. Также возможны большие погрешности из-за трудности оптимизации эксперимента. На нашем модифицированном эллипсометре возможен другой вариант решения задачи. Выбором определенной ориентации оптической оси анизотропной отражающей системы относительно плоскости падения (симметричные ориентации) достигается равенство нулю недиагональных элементов матрицы отражения. Такая методика для определения четырех параметров отражающей системы была экспериментально реализована в работах [7-9]. В настоящей работе развитые подходы в работах $[1,9,12]$ реализуются применительно к исследованию тетрабората стронция, активированного ионами иттербия, который считается аморфным стеклом и которое, казалось бы, не должно обладать оптической анизотропией. Забегая вперед, отметим, что даже для такого объекта была обнаружена и измерена анизотропия, соизмеримая с инструментальными погрешностями угломерных устройств эллипсометра.

\section{Новые подходы и роль измерительных зон эллипсометрии в исследовании слабоанизотропных систем}

В обычной схеме измерения на эллипсометре в качестве исследуемого объекта используется тщательно отполированная с двух сторон плоскопараллельная пластинка из прозрачного материала, проводятся обычные эллипсометрические измерения от каждой стороны этой пластинки, как показано на рис. 1. При отражении от верхней грани плоскопараллельной пластинки (геомет- 


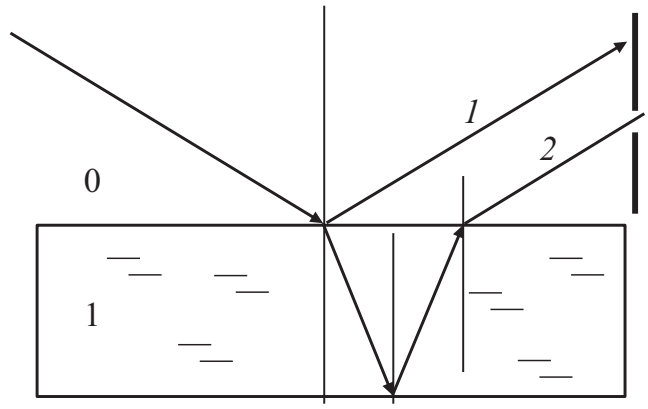

Pис. 1. Схема измерения для двух геометрий отражения $M 1$ и $M 2$ : в геометрии $M 1$ вырезается пучок 2, в геометрии $M 2$ вырезается пучок 1 .

рия $M 1)$ можно использовать выражение $[1,10,13,14]$

$$
N=n-i k=N_{0} \operatorname{tg} \varphi\left[1-\frac{4 \rho}{(1+\rho)^{2}} \sin ^{2} \varphi\right]^{1 / 2},
$$

где $\varphi-$ угол падения светового пучка на отражающую систему, $N_{0}$ - показатель преломления внешней среды (для воздуха его принято считать равным единице), $n$ и $k$ - показатели преломления и затухания образца соответственно.

Как было показано в работах [10-12], при определении показателя преломления главным источником погрешности является неточная установка угла падения. Из уравнения (2) для однородной полубесконечной среды зависимость погрешности $d N$ комплексного показателя преломления $N$ от угла падения $\varphi$ определяется [12] из выражения

$$
d N=\left[N\left(\operatorname{tg} \varphi+\frac{2}{\operatorname{tg} \varphi}\right)-\frac{N_{0}^{2} \operatorname{tg} \varphi}{N}\right] d \varphi
$$

Из выражения (3) видно, что погрешность увеличивается с ростом комплексного показателя преломления подложки $N$. Из этого выражения легко определить угол падения, при котором погрешность $d N$ минимальна для любого конкретного значения комплексного показателя преломления $N$. Например, для $n=1.58$ вблизи угла падения $65^{\circ}$ наблюдается погрешность $d n$ порядка $10^{-3}$, и этот угол является оптимальным для определения $n$. Такие углы легко найти для любого показателя преломления. Заметим, что с ростом показателя преломления уменьшается угол падения, при котором погрешность минимальна для данного конкретного значения показателя преломления. Как было подчеркнуто в работе [1], вблизи угла Брюстера резко увеличивается не только чувствительность эллипсометрических углов к измеряемым параметрам отражающей системы, но и их погрешность. Для показателя преломления $n=1.58$ угол Брюстера составляет 57.69 ${ }^{\circ}$. Как было показано в работе [12], для диэлектрических подложек вблизи угла Брюстера эллипсометрический угол $\Psi$ стремится к нулю, и резко растет погрешность определения параметра $\Delta$. Ранее было доказано, что для определения объемного значения показателя преломления $n_{b}$ прозрачной среды угол Брюстера не является оптимальным [12].

Для геометрии $M 2$ (при отражении от нижней грани) было получено следующее выражение [1]:

$$
N_{1}=f \sqrt{\frac{N_{0}^{2}-a f^{2}}{N_{0}^{2}-f^{2}}} ; f=N_{0} \sin \varphi ; a=\frac{4 t \rho_{1}}{\left(1+t \rho_{1}\right)^{2}},
$$

где $\rho_{1}-$ комплексный амплитудный коэффициент отражения, определяемый из выражения

$$
\rho_{1}=\operatorname{tg} \Psi_{1} \exp \left(i \Delta_{1}\right)
$$

Здесь $\Psi_{1}$ и $\Delta_{1}$ - эллипсометрические параметры, экспериментально измеряемые для геометрии отражения (M2). Использование выражения (4) позволяет измерять $n_{b}$ (объемное значение показателя преломления) плоскопараллельной пластинки. Параметр $t$ является функцией показателя преломления внешней среды (воздуха) и показателя преломления подложки. В работах $[13,14]$ предполагалось, что этот параметр известен или предварительно вычислен. Для рассматриваемой нами плоскопараллельной пластинки коэффициент $t$ может быть определен экспериментально [1]: $t=\rho / \rho_{1}$.

При геометрии $M 2$ световой пучок проходит дважды через плоскопараллельную пластинку (рис. 1), поэтому возможно обнаружение неоднородности не только на границах раздела, но и в объеме исследуемого объекта. Так как в данном случае световой пучок дважды проходит объем пластинки, вклад в формирование пучка от поверхностного слоя (ПС) существенно меньше, чем от объема материала. В данном случае имеем $t_{e}$ (экспериментальное) и $t_{c}$ (расчетное) значения коэффициентов. В идеале они должны совпадать. В работе [1] было продемонстрировано, что $t_{e}$ и $t_{c}$ для плавленого кварца полностью совпадают, и результаты измерений для геометрии $M 1$ и $M 2$ идентичны.

При исследовании анизотропных объектов методом эллипсометрии возникают не только добавочные возможности, но и сложности. При отражении от анизотропной системы возможно появление недиагональных элементов матрицы отражения, следовательно, имеются три уравнения, что позволяет определять до шести параметров отражающей системы. Но как было отмечено выше, в этом случае решение как прямой, так и обратной задачи эллипсометрии весьма громоздкое и трудоемкое. Возможны также большие погрешности. Для исследования анизотропных систем ключевым моментом является точная юстировка эллипсометра, включая и калибровку угломерных устройств (лимба) угла падения света на образец, особенно при исследовании слабой анизотропии. Следует подчеркнуть, что в тщательно отъюстированном приборе для изотропных отражающих систем выполняются все соотношения измерительных зон эллипсометра [14]. В модифицированном эллипсометре 
ЛЭФ-2 эллипсометрические углы $\Psi, \Delta$, измеренные в четырех измерительных зонах, равносильны четырем независимым измерениям. Уместно напомнить, что в работе [14] с помощью соотношений измерительных зон была обнаружена слабая оптическая анизотропия на поверхности тонких пленок никеля.

\section{Юстировка эллипсометра в исследовании анизотропных отражающих систем}

Как отмечено выше, точная юстировка эллипсометра для исследования анизотропных систем является ключевым моментом. В настоящей работе мы вынуждены в общих чертах затрагивать этот вопрос (более подробно см. ниже). Следует заметить, что точная юстировка необходима для всех типов эллипсометра, включая современные автоматические. Юстировку эллипсометра можно разделить на три части. Первая механическая юстировка, которая должна проводиться в промышленных условиях или в процессе изготовления эллипсометров [10]. Вторая - оптическая юстировка или калибровка лимбов угломерных устройств поляризационных элементов (поляризатора, анализатора и компенсатора) относительно плоскости падения света на образец. Третья - это разбиение на измерительные зоны [11]. Следует подчеркнуть, что все эти три части взаимосвязаны. Часто полагают, что механическая юстировка проведена идеально, и в основном ведутся обсуждения методов оптической юстировки [15-17].

Следует уточнить, что при оптической юстировке эллипсометра основная задача сводится к определению плоскости падения на лимбах угломерных устройств поляризационных элементов эллипсометра (поляризатора, анализатора и компенсатора), и при этом для всех углов падения положение плоскости падения не должно изменяться. Это требование обеспечивается механической юстировкой эллипсометра, поэтому практически всегда полагается, что оно выполнено в промышленных условиях безукоризненно. Практика показывает, что не на всех промышленных эллипсометрах выполнено это требование. Кроме того, при изменении температуры окружающей среды или изменении моды лазерного излучения не исключена вероятность, что изменяется положение плоскости падения. Поэтому важной задачей является контроль сохранения плоскости падения в процессе каждодневной эксплуатации эллипсометра. Плоскости падения отвечает то показание на лимбе поляризатора $P_{0}$, при котором ось пропускания поляризатора совпадает с плоскостью падения. На лимбе анализатора фиксируются показания $A_{0}$ и $A_{0}+90$. В первом случае ось пропускания анализатора перпендикулярна плоскости падения, во втором - совпадает с ней [9].

Оптическую юстировку эллипсометра можно проводить несколькими способами. Один из старых способов описан в работах $[9,10,16,18]$. Он основан на том, что при отражении света от изотропной подложки линейная поляризация сохраняется, когда вектор светового поля лежит в плоскости падения $(p)$ или перпендикулярен $(s)$ ей. Процесс юстировки заключается в поиске положения самого глубокого минимума. Однако этот метод неудобный и трудоемкий. В этом методе возможны самые различные погрешности. Например, если подложка прозрачна, то возможно сохранение линейной поляризации и для положений вектора электрического поля, отличных от $s$ и $p$. Возможны и другие погрешности при использовании данного способа, например при наличии слабой оптической анизотропии $[10,16]$. При отражении от металлической поверхности возникают неоднородные волны, которые вносят добавочные сложности и погрешности.

Другой способ - использование угла Брюстера при отражении от поверхности диэлектрика $[9,10,16,18]$. В настоящее время можно подготовить поверхность плавленого кварца, которая работает как идеально гладкая, и отраженный свет при угле Брюстера будет полностью поляризованным [1]. Предполагается, что в этом методе точно устанавливается угол Брюстера и поворотом поляризатора достигается полное гашение. Достигнутое значение показания на лимбе угломерного устройства поляризатора является искомым значением $P_{0}$, которое соответствует совпадению плоскости падения и оси пропускания поляризатора. В этом методе предварительно из эллипсометра убираются компенсатор и анализатор. После определения значения $P_{0}$ плечи эллипсометра устанавливаются в горизонтальном положении (т. е. на просвет). В плече анализатора устанавливается анализатор, и его поворотом на выходе эллипсометра достигается максимальное гашение. Достигнутое значение показания на лимбе угломерного устройства анализатора является искомым значением $A_{0}$, которое соответствует совпадению оси пропускания анализатора с плоскостью, перпендикулярной плоскости падения [9]. Далее в схему эллипсометра вводится компенсатор. В положениях поляризатора и анализатора, соответствующих показаниям $P_{0}$ и $A_{0}$, поворотом компенсатора достигается максимальное гашение света на выходе эллипсометра. Полученное значение показания на угломерном лимбе компенсатора является параметром $C_{0}$. Это означает, что „быстрая“ или „медленная“ ось компенсатора совпадает с плоскостью падения. Следует подчеркнуть, что в этом методе скрыта некоторая неопределенность или неоднозначность, которая неочевидна. Действительно, если даже предположить, что точно известен показатель преломления отражающей системы (подложки) и соответственно значение угла Брюстера, это не означает, что точно установлен угол падения и соответственно определено точное положение плоскости падения на лимбе угломерного устройства поляризатора. Наоборот, если точно известен показатель преломления идеально гладкого диэлектрика, возникает возможность откалибровать (или контролировать) лимб угломерного устройство угла падения на эллипсометре. 


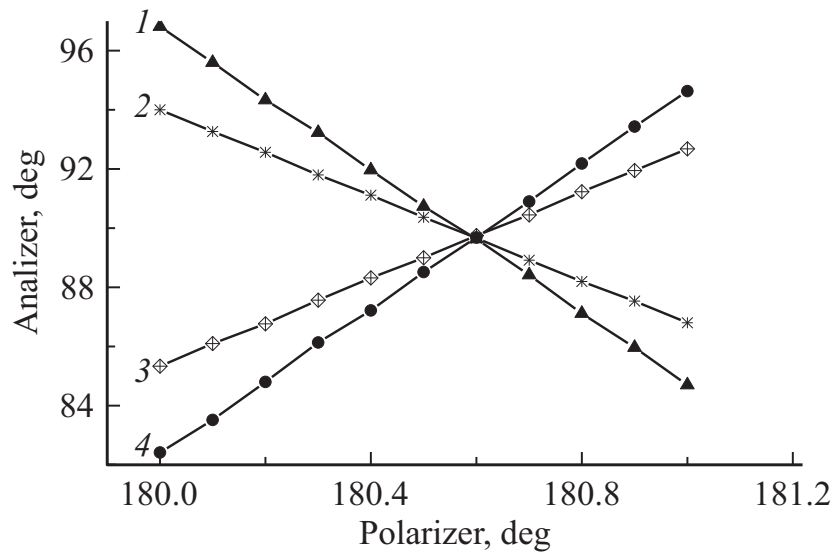

Рис. 2. Юстировка или калибровка угломерных устройств (лимбов) поляризатора и анализатора методом скрещенных поляризаторов в отсутствие компенсатора в схеме эллипсометра. По обеим осям координат углы в градусах. Зависимость $A(P)$ при различных углах падения светового пучка на диэлектрик: 63 (1), 59 (2), 79 (3), 75 (4). Поляризатор поворачивается на значения через $0.1^{\circ}\left(6^{\prime}\right)$, затем поворотом анализатора находятся соответствующие положения максимального гашения. Все четыре кривые пересекаются в одной точке с координатами: $P_{0}=180^{\circ} 36^{\prime}$ и $A_{0}=89^{\circ} 42^{\prime}$.

Еще один распространенный метод - это метод „скрещенных“ поляризаторов в отсутствие компенсатора в схеме эллипсометра при отражении света от диэлектрика $[10,11,15-17,19]$. Суть метода заключается в следующем. Из эллипсометра удаляется компенсатор. Угол падения эллипсометра устанавливается меньше угла Брюстера примерно на $5^{\circ}-10^{\circ}$ для выбранного диэлектрика. Достигается положение гашения на угломерных лимбах поляризатора и анализатора. Затем поляризатор поворачивается, например, на $3^{\circ}$ или $5^{\circ}$ и только поворотом анализатора достигается гашение на выходе анализатора. Эта процедура повторяется для нескольких значений через каждые $3^{\circ}$ или $5^{\circ}$. Строится кривая зависимости $A(P)$. Аналогичная кривая строится для угла падения, превышающего угол Брюстера на $5^{\circ}-10^{\circ}$. Пересечение этих кривых дает искомые углы $P_{0}$ и $A_{0}$. Для достоверности обычно строят 3 или 4 кривые. Если поляризаторы являются идеальными (т.е. если остаточная эллиптичность пренебрежимо мала), то все кривые пересекаются в одной точке. Точно так же можно проводить вышеописанные процедуры для построения зависимости $P(A)$. В качестве примера на рис. 2 приведены кривые $A(P)$, полученные на модифицированном эллипсометре ЛЭФ-2. Кривые могут пересекаться в разных точках в случае наличия погрешности в механической юстировке.

Наиболее удобным, быстрым и более универсальным является метод, предложенный в работе [20]. Этот метод позволяет проводить оптическую юстировку в рабочем положении эллипсометра, не убирая из прибора ни одного поляризационного элемента. Метод важен для повседневного контроля юстировки эллипсометра. Эту юстировку можно проводить при любом угле падения, и совпадение параметров юстировки $\left(P_{0}, A_{0}, C_{0}\right)$ для всех углов падения свидетельствует о постоянстве плоскости падения при изменении угла падения. Таким образом, при данном методе, кроме перечисленных достоинств, проводится также проверка механической юстировки эллипсометра.

Следует отдать должное Аюпову Б.М. [16], который всесторонне анализировал достоинствоа и недостатки метода, предложенного в работе [20]. Необходимо отметить, что в работах $[21,22]$ были подробно изложены особенности применения предложенного в работе [20] метода при наличии и отсутствии компенсатора в схеме эллипсометра. Тем не менее заслуживает внимания работа [16], особенно для широкого круга специалистов, для быстрого освоения и использования в своей практике метода, предложенного в работе [20], позволяющего также избежать специалистам возможные ошибки. Более того, Б.М. Аюпов, детально анализируя методы оптической юстировки, предложил новый подход для обнаружения оптической анизотропии [15-17,19]. Заметим, что подход для обнаружения слабой оптической анизотропии, развитый Б.М. Аюповым в работах $[15-17,19]$, к сожалению, не позволяет обнаружить слабую оптическую анизотропию в нашем объекте тетраборате стронция, активированном ионами иттербия, как и в некоторых других объектах, обладающих слабой оптической анизотропией.

\section{Эксперимент}

Все эллипсометрические измерения проводились на

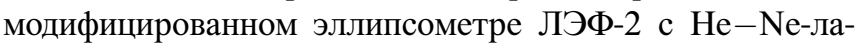

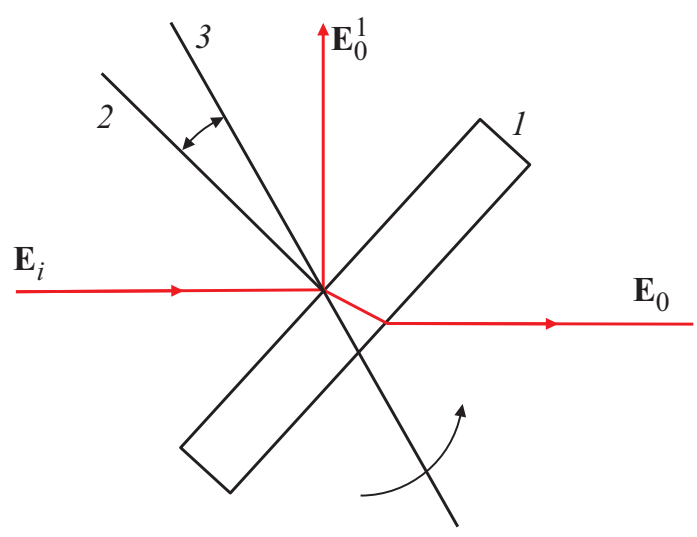

Рис. 3. ОЭ (1) - оптический элемент эллипсометра (поляризатор, анализатор или компенсатор); $\mathbf{E}_{i}, \mathbf{E}_{0}^{1}$ и $\mathbf{E}_{0}-$ падающий, отраженный и проходящий световые векторы (пучок); НП (2) — нормаль к поверхности ОЭ; ОВ (3) ось вращения ОЭ. Необходимо, чтобы НП и ОВ совпадали. Для наглядности все углы (угол наклона ОЭ, между НП и $\mathrm{OB}$, углы падения, отражения и преломления) представлены в увеличенном виде. 
Таблица 1. Результаты усредненных стандартных многоугловых измерений на двух образцах

\begin{tabular}{|c|c|c|c|c|c|c|c|c|}
\hline \multirow{2}{*}{$\Phi, \operatorname{deg}$} & \multicolumn{4}{|c|}{ Образец 1} & \multicolumn{4}{|c|}{ Образец 2} \\
\hline & $\Psi, \operatorname{deg}$ & $\Delta, \operatorname{deg}$ & $n$ & $k$ & $\Psi, \operatorname{deg}$ & $\Delta, \operatorname{deg}$ & $n$ & $k$ \\
\hline 70 & 19.129 & 4.458 & 1.567 & -0.068 & 18.667 & 3.208 & 1.584 & -0.049 \\
\hline 65 & 11.721 & 8.133 & 1.565 & -0.068 & 11.133 & 5.25 & 1.586 & -0.046 \\
\hline 60 & 4.242 & 25.008 & 1.563 & -0.065 & 3.583 & 19.100 & 1.591 & -0.047 \\
\hline 55 & 4.108 & 156.575 & 1.569 & -0.069 & 4.554 & 165.433 & 1.588 & -0.046 \\
\hline 50 & 11.388 & 172.142 & 1.567 & -0.064 & 11.763 & 174.967 & 1.59 & -0.043 \\
\hline 45 & 18.279 & 175.767 & 1.566 & -0.062 & 18.642 & 177.042 & 1.586 & -0.044 \\
\hline
\end{tabular}

Таблица 2. Результаты усредненных стандартных измерений на двух образцах для двух ориентаций и двух геометрии отражения

\begin{tabular}{c|c|c|c|r|r}
\hline \multirow{2}{*}{ Схема } & \multirow{2}{*}{$\begin{array}{c}\text { Средние } \\
\text { значения }\end{array}$} & \multicolumn{2}{|c|}{ Образец 1 } & \multicolumn{2}{|c}{ Образец 2 } \\
\cline { 3 - 6 } & параллельно & перпендикулярно & параллельно & перпендикулярно \\
\hline \multirow{2}{*}{$M 1$} & $\Psi$, deg-min & $11-57$ & $12-11$ & $11-05$ & $11-04$ \\
& $\Delta$, deg-min & $10-04$ & $8-01$ & $4-01$ & $4-25$ \\
\hline \multirow{2}{*}{$M 2$} & $\Psi$, deg-min & $14-09$ & $14-51$ & $14-32$ & $14-29$ \\
& $\Delta$, deg-min & $26-44$ & $9-27$ & $33-32$ & $22-57$
\end{tabular}

зером на длине волны $\lambda=632.8 \mathrm{~nm}$. В отличие от стандартного эллипсометра ЛЭФ-2, где угол падения изменяется в интервале $50^{\circ}-90^{\circ}$, в этом модифицированном приборе угол падения изменяется от $45^{\circ}$ до $90^{\circ}$.

В данном приборе используется идеальное четвертьволновое фазосдвигающее устройство $\left(T_{C}=1\right.$, $\left.\delta_{C}=90^{\circ}\right)$ [23]. При изменении температуры окружающей среды в пределах $\pm 3^{\circ} \mathrm{C}$ у этого фазосдвигающего устройства параметры практически не изменяются. Параметр $T_{C}$ характеризует отношение амплитудных (по модулю) коэффициентов пропускания вдоль медленной и быстрой осей компенсатора. Параметр $\delta_{C}-$ сдвиг фазы между компонентами вектора напряженности электрического поля, параллельного этим осям, приобретаемый падающей световой волной на выходе пластинки. У поляризатора и анализатора малая эллиптичность для обоих поляризаторов (призмы Глана-Фуко) не превышала $3^{\prime}$. Все поляризующие оптические элементы в эллипсометре установлены таким образом, что ось вращения и нормаль к поверхности совпадают, а направление распространения светового пучка на незначительный угол отклонено от нормали [12] (рис. 3).

Измерения в четырех неэквивалентных зонах на этом приборе для изотропных или симметричных ориентаций любой отражающей системы равносильны четырем независимым измерениям. Кроме того, при повороте образца вокруг нормали к поверхности положение пятна от пучка на образце не изменяется, т.е. измерения проводятся на строго заданной точке на поверхности образца.

Исследовалось стронций-боратное стекло, состав которого определялся следующим соотношением компонентов: $32 \mathrm{SrO}-65 \mathrm{~B}_{2} \mathrm{O}_{3}-3 \mathrm{Yb}_{2} \mathrm{O}_{3}$ или $\mathrm{Yb}: \mathrm{SBO}$. Стекло
Таблица 3. Результаты расчета показателей преломления $n_{o}$ и $n_{e}$ и коэффициентов $t_{e}, t_{c}$

\begin{tabular}{c|c|c|c|c}
\hline \multirow{2}{*}{ Параметр } & \multicolumn{2}{|c|}{ Образец 1 } & \multicolumn{2}{c}{ Образец 2 } \\
\cline { 2 - 5 } & $n_{o}$ & $n_{e}$ & $n_{o}$ & $n_{e}$ \\
\hline$n$ & 1.555 & 1.544 & 1.591 & 1.592 \\
$t_{c}$ & 0.760 & 0.764 & 0.746 & 0.745 \\
$t_{e}$ & 0.834 & 0.815 & 0.768 & 0.755 \\
$\delta t$ & 0.64 & 0.51 & 0.22 & 0.10
\end{tabular}

было получено стандартным методом сплавления [3]. Оно было тщательно отполировано с двух сторон. Часть образцов после синтеза подвергалась термообработке при температуре значительно ниже стеклования $\left(600-800^{\circ} \mathrm{C}\right)$ для удаления областей с оптической дисторсией. Плотность синтезированного стекла, равная $3.1 \mathrm{~g} / \mathrm{cm}^{3}$, была определена гидростатическим методом. Как и ожидалось, она была ниже плотности кристалла $\mathrm{SrB}_{4} \mathrm{O}_{7}$, которая равна $3.99 \mathrm{~g} / \mathrm{cm}^{3}$ [24]. Измерения на рентгеновских приборах не позволили обнаружить наличие анизотропии. Также при стандартных эллипсометрических измерениях анизотропия не была обнаружена. Только новый подход, развитый в работах $[1,7-9]$, позволил обнаружить анизотропию. Результаты экспериментальных данных для двух образцов представлены в двух таблицах.

В табл. 1 приведены результаты стандартных многоугловых эллипсометрических измерений для двух образцов. Толщина образцов $d=1.4 \mathrm{~mm}$, длина $l=25 \mathrm{~mm}$. Из сравнения результатов для образцов 1 и 2 видно, что показатель затухания $k$ для первого образца больше, чем 

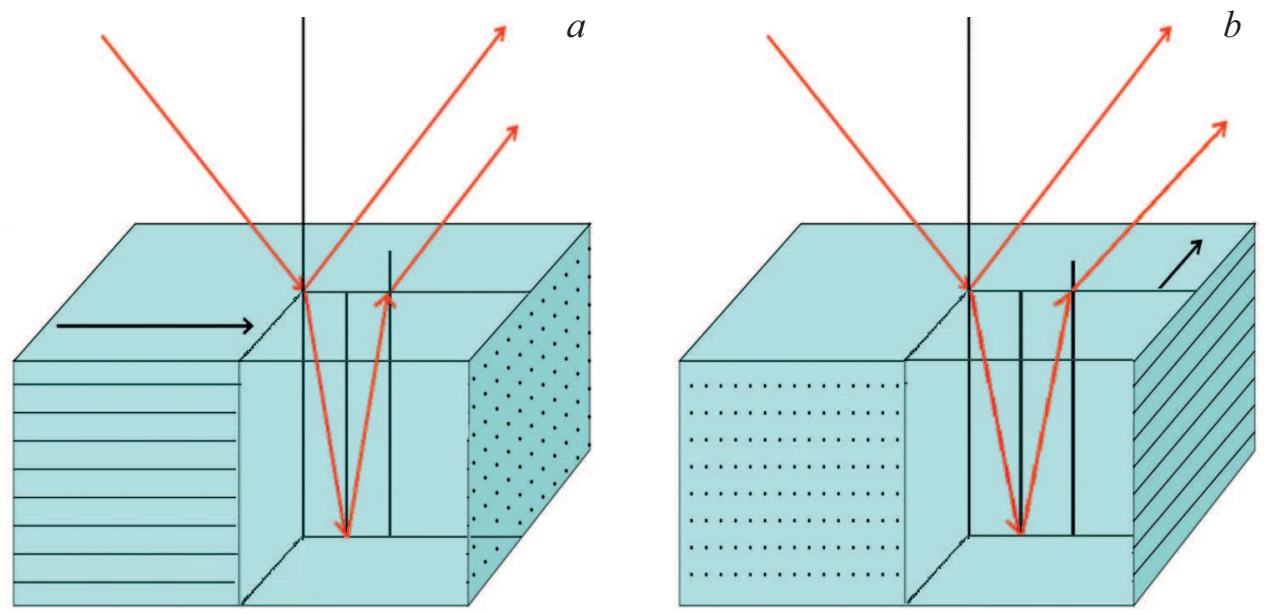

Рис. 4. Принципиальная схема и ход лучей при измерении эллипсометрических параметров $\Psi$ и $\Delta$ образца в геометрии $M 2$, когда оптическая ось параллельна $(a)$ и перпендикулярна $(b)$ плоскости падения.

для образца 2. Как было показано в работе [1], отличие показателя затухания $k$ от нуля обусловлено возможным наличием пленки на поверхности или неоднородностями в объеме пластинок, так как они являются прозрачными для данной зондирующей электромагнитной волны $(\lambda=632.8 \mathrm{~nm})$. Заметим, что на образце 2 был проведен отжиг, а на образце 1 нет. Следовательно, отжиг улучшает характеристику стекла.

В табл. 2 представлены усредненные значения результатов измерений $\Psi$ и $\Delta$ для обоих образцов в геометрии $M 1$ и $M 2$. Ориентация оптической оси была определена в геометрии $M 2$ по методике, изложенной в работах [7-9,12]. Из табл. 2 видно, что отличие $\Psi, \Delta$ для двух ориентаций (симметричных, т. е. когда недиагональные матрицы отражения Джонса равны нулю) по геометрии $M 1$ существенно меныше, чем по геометрии $M 2$. Особенно это заметно для второго образца, где разность $\Psi$ всего одна угловая минута. Это значение находится в пределах инструментальной погрешности для эллипсометров серии ЛЭФ-2, ЛЭФ-3 и других.

Для ясности необходимо более подробно остановиться на предмете определения и установления оптических осей в плоскости падения для геометрии отражения $M 1$ и $M 2$ на образцах. Как было отмечено выше, для геометрии $M 1$ определение ориентации оптических осей по методике, изложенной в работах [7-9,12], не подходит, так как анизотропия слабая, и при повороте образца вокруг нормали к его поверхности сигнал практически не изменяется на выходе анализатора. Однако в геометрии $M 2$ ориентация оптической оси достаточно легко и точно определяется по методике, изложенной в работах [7-9,12]. Здесь уместно вспомнить суть методики, изложенной в работах [7-9,12], для определения и установления ориентации оптической оси в плоскости падения или перпендикулярно ей. Она заключается в следующем.
Перед началом измерений оптическая ось образца устанавливается в плоскости падения или перпендикулярно ей. Для этого на лимбах эллипсометра выбираются положения $P_{0}, A_{0}, C_{0}$, и поворотом образца вокруг нормали к поверхности достигается максимальное гашение на выходе. Следует подчеркнуть, что при геометрии отражения $M 2$ малейший выход оптической оси образца из плоскости падения приводит к резкому росту сигнала на выходе эллипсометра. Поэтому в этом случае с достаточно высокой точностью устанавливается оптическая ось в плоскости падения. На рис. 4 представлена схема измерений эллипсометрических углов $\Psi$ и $\Delta$ для геометрии отражения $M 1$ и $M 2$ применительно к нашим объектам исследования.

В табл. 3 представлены результаты расчета для значений показателей преломления $n_{o}$ и $n_{e}(n)$ по алгоритмам, изложенным в работах [7-9,12]. Там же представлены значения $t_{c}$ и $t_{e}$ для обоих образцов в рамках изотропной отражающей системы. Видно, что значения $t_{c}$ и $t_{e}$ существенно отличаются, что также подтверждает наличие анизотропии. Мнимая часть показателей преломления отброшена.

Итак, в стекле, полученном на основе кристалла тетрабората стронция, активированного ионами иттербия, обнаружено наличие анизотропии и определены показатели преломления обыкновенной и необыкновенной волн. Расчет проведен в предположении оптически одноосной анизотропии с использованием алгоритмов, изложенных в работах [7-9,12].

\section{Заключение и выводы}

Слабую оптическую анизотропию по результатам стандартных многоугловых или других прямых эллипсометрических измерений обнаружить практически невозможно. Новый эллипсометрический метод позволяет обнаружить наличие анизотропии и измерить пока- 
затели преломления обыкновенной и необыкновенной волн. На примере исследования синтезированного стекла на основе кристалла тетрабората стронция, который активирован ионами иттербия, показана возможность этого метода. В этом стекле обнаружена анизотропия и определены показатели преломления обыкновенной и необыкновенной волн. Плотность синтезированного стекла, равная $3.1 \mathrm{~g} / \mathrm{cm}^{3}$, была определена гидростатическим методом. Как и ожидалось, она была ниже плотности кристалла $\mathrm{SrB}_{4} \mathrm{O}_{7}$, которая равна $3.99 \mathrm{~g} / \mathrm{cm}^{3}$.

Предложенная методика позволяет обнаружить анизотропию в пределах инструментальной погрешности серийных эллипсометров. Для исследования и обнаружения слабой оптической анизотропии точная юстировка эллипсометра и выполнение соотношений измерительных зон эллипсометра являются ключевыми моментами.

\section{Конфликт интересов}

Автор заявляет, что у него нет конфликта интересов.

\section{Список литературы}

[1] Хасанов T.X. // Опт. и спектр. 2015. Т. 118. № 4. С. 684; Khasanov T.Kh. // Opt. Spectrosc. 2015. V. 118. N 4. P. 655.

[2] Сопинский Н.В. // Опт. и спектр. 2017. Т. 123. В. 5. С. 764; Sopinskii N.V. // Opt. Spectrosc. 2017. V. 12. N 5. P. 778.

[3] Дмитрук Л., Петрова О., Попова А., Шукшин В. // Труды ИОФАН. 2008. Т. 64. С. 49.

[4] Liaolin Zhang, Yu Xia, Xiao Shen, Runlan Yang, Wei Wei // Opt. Mater. 2018. V. 75. P. 1.

[5] Зубрилов А.С., Мельник Ю.В., Николаев А.Е.,. Якобсон М.А, Нельсон Д.К., Дмитриев В.А. // ФТП. 1999. Т. 33. № 10. C. 1173.

[6] Туркин А.Н. // Компоненты и технологии. 2011. № 5. С. 6.

[7] Любинская Р.И., Мардежсов А.С., Свиташев К.К., Хасанов T. // Опт. и спектр. 1985. Т. 59. В. 2. C. 353; Lyubinskaya R.I, Mardezhov A.S., Svitashev K.K, Khasanov T. // Opt. Spektrosc. 1985. V. 59. N 2. P. 353.

[8] Любинская Р.И., Мардежсов А.С., Свиташев К.К., Хасанов Т.X. // Опт. и спектр. 1988. Т. 65. В. 3. C. 632; Lyubinskaya R.I.,. Mardezhov A.S., Svitashev K.K., Khasanov T. // Opt. Spektrosc. 1988. V. 65. N 3. P. 632.

[9] Мутилин С.В., Хасанов Т. // Опт. и спектр. 2008. Т. 105. № 3. C. 512; Mutilin S.V., Khasanov T. // Opt. Spectrosc. 2008. V. 105. N 3. P. 461.

[10] Аззам Р., Башара Н. Эллипсометрия и поляризованный свет. М., 1981. 583 c.

[11] Ржанов А.В., Свиташев К.К, Семененко Л.В., Семененко А.И., Соколов В.К. Основы эллипсометрии. Новосибирск, 1979. $422 \mathrm{c.}$

[12] Хасанов T.X. // Фундаментальные проблемы современного материаловедения. 2011. Т. 8. № 3. С. 62.

[13] Григорьева Т.И., Хасанов Т.Х. // Опт. и спектр. 2010. T. 108. № 4. C. 629; Grigor'eva T.I., Khasanov T.Kh. // Opt. Spectrosc. 2010. V. 108. N 4. P. 591.

[14] Григорьева Т.И., Хасанов Т.Х. // Опт. и спектр. 2012. T. 112. № 5. C. 851; Grigor'eva T.I., Khasanov T.Kh. // Opt. Spectrosc. 2012. V. 112. N 5. P. 796.
[15] Аюпов Б.М.,. Козлова Н.А. // Оптический журнал. 2006. T. 73. № 3. C. 72; Ayupov B.M., Kozlova N.A. // J. Opt. Technol. 2006. V. 73. N 3. P. 212.

[16] Аюпов Б.М. // Оптический журнал. 1997. Т. 643. № 12. C. 103; Ayupov B.M. // J. Opt. Technol. 1997. V. 64. N 12. P. 1166.

[17] Ayupov B.M. // Optik. 1998. V. 109. N 4. P. 145.

[18] McGrackin F.L., Passaglia E., Stromberg R.R., Steinberg H.L. // J. Res. Natl. Bur. Std. 1963. V. 67A. P. 363.

[19] Аюпов Б.М. // Автометрия. 1997. № 1. С. 95; Ayupov B.M. // Optoelectronics, Instrumentation and Data Processing. (Avtometriya). 1997. N 1. P. 85.

[20] Свиташев К.К., Хасанов Т. // Опт. и спектр. 1986. Т. 61. B. 2. C. 399; Svitashev K.K., Khasanov T. // Opt. Spektrosc. 1986. V. 60. N 2. P. 399.

[21] Хасанов T. // Препринт ИФП СО АН СССР. 7-90. Новосибирск, 1990. 32 с.

[22] Хасанов T. // Автометрия. 1997. В. 1. С. 81; Hasanov T. // Optoelectronics, Instrumentation and Data Processing. (Avtometriya). 1997. N 1. P. 73.

[23] Хасанов T.X. Фазосдвигающее устройство. Патент РФ № 2308066.

[24] Pan F., Shen G., Wang R. et al. // J. Crystal Growth. 2002. V. 241. P. 108. 